\title{
Commentary: Mismatch Repair Deficiency and Microsatellite Instability in Triple-Negative Breast Cancer: A Retrospective Study of 440 Patients
}

\author{
Konstantinos Venetis ${ }^{1,2}$, Nicola Fusco ${ }^{1,2 *}$ and Elham Sajjadi ${ }^{1,2}$ \\ ${ }^{1}$ Division of Pathology, IEO, European Institute of Oncology IRCCS, Milan, Italy, ${ }^{2}$ Department of Oncology and \\ Hemato-Oncology, University of Milan, Milan, Italy
}

Keywords: triple-negative breast cancer (TNBC), mismatch repair (MMR), microsatellite instability (MSI), biomarkers, testing methods

\section{A Commentary on}

\section{OPEN ACCESS}

Edited by:

Todd M. Pitts,

University of Colorado, United States

Reviewed by:

Jennifer Diamond,

University of Colorado Cancer Center,

United States

${ }^{*}$ Correspondence:

Nicola Fusco

nicola.fusco@unimi.it

Specialty section:

This article was submitted to Molecular and Cellular Oncology,

a section of the journal

Frontiers in Oncology

Received: 02 July 2021 Accepted: 13 September 2021 Published: 29 September 2021

Citation:

Venetis K, Fusco N and Sajjadi E (2021) Commentary: Mismatch Repair

Deficiency and Microsatellite Instability

in Triple-Negative Breast Cancer: A Retrospective Study of 440 Patients.

Front. Oncol. 11:735476.

doi: 10.3389/fonc.2021.735476
Mismatch Repair Deficiency and Microsatellite Instability in Triple-Negative Breast Cancer: A Retrospective Study of $\mathbf{4 4 0}$ Patients by Ren X-y, Song Y, Wang J, Chen L-y, Pang J-y, Zhou L-r, Shen S-j, Cao X, Wang Y-x, Shao M-m, Liang Z-y, Sun Q and Wu H-w (2021). Front. Oncol. 11:570623. doi: 10.3389/fonc.2021.570623

\section{INTRODUCTION}

The mismatch repair (MMR) system maintains the genomic stability through the correction of base mispairing generated during DNA replication (1). Its deficiency has a relevant role in the tumorigenesis and tumor progression of a subset of breast cancers (2).

In an interesting study, Ren and collaborators (3) focus the attention on triple-negative breast cancers (TNBC). Using MMR immunohistochemistry (IHC) and microsatellite instability (MSI) PCR on a retrospective cohort of 440 patients, the Authors found only 1 (0.2\%) MMR-deficient (dMMR) case, showing loss of MSH2 alone and low-frequency MSI (MSI-L). No MSI-high (MSI-H) tumors were observed, although overall 14 (7.2\%) samples were MSI-L. The Authors confirm the low incidence of dMMR/MSI-H (4) and the high rate of discrepancy between MMR IHC/MSI PCR in TNBC (5). Finally, their analyses revealed no significant associations between MSI-L and other clinicopathological and prognostic features.

The topic is of great importance considering the growing interest on the implementation of consistent MMR testing for prognostication, immune checkpoints inhibitors (ICI) prediction, and identification of therapy resistance/susceptibility in both adjuvant and neoadjuvant settings (6-8). To date, in the neoadjuvant setting, several clinical trials have examined the efficacy of programmed death-1/programmed death-ligand 1 (PD-1/PD-L1) blockade in early high-risk TNBC (9-11). The results of the KEYNOTE-522 study have recently led to the approval of pembrolizumab in combination with chemotherapy for patients with locally recurrent unresectable or metastatic TNBC whose tumors express PD-L1 with combined positive score (CPS) $\geq 10$ (10). Despite these 
remarkable achievements, additional biomarkers would be helpful in this setting. Therefore, this elegant work by Ron et al. is an excellent opportunity to reflect on the possibilities and challenges of MMR analysis for patients with TNBC.

\section{FREQUENCY AND SPECTRUM OF MISMATCH REPAIR ALTERATIONS IN TNBC}

Types of MMR alterations described in TNBC, include gene mutations, hypermethylation, RNA downregulation, and alterations in the expression patterns of the protein complexes $(5,12-16)$. The actual frequency of dMMR in TNBC, however, is controversial, since MMR mutations are reported in $2 \%$ of cases, while an impaired protein expression seems to be more frequent $(15,17,18)$, probably due to post-transcriptional modifications (Figure 1). Interestingly, dMMR TNBC often present a single protein loss (19), as also noted by Ren et al.

\section{THE RATIONALE FOR MMR CLINICAL TESTING IN TNBC}

The previously reported significant prognostic role of dMMR in TNBC $(6,15)$ has not been confirmed by Ren et al. because they found only $1 \mathrm{IHC} \mathrm{dMMR}$ and no MSI-H cases. In this respect, a study by our group focusing on MMR patterns of expression showed a better prognosis for TNBC tumors with MMR proteins perturbations (5). Regarding the predictive role, although data on MMR alterations in TNBC are still being generated (20), the existing evidence is limited and therefore, further studies to establish its clinical value are expected. Hence, only a few TNBC were included in the basket trials that led to the ICI MMR-based histology-agnostic approval (21). Furthermore, the notion that relates the sensitivity to ICI to the adaptative immune response against neo-antigens, generated by super-mutator cancer cells, is another facet that needs further clarification in TNBC (22). Indeed, the tumor mutation burden observed in dMMR TNBC is overall lower than in other types of dMMR cancers, albeit significantly higher than in hormone receptor (HR)-positive breast cancers $(2,23)$. The interaction between MMR and other immune-related biomarkers in TNBC could be explored in the near future to improve a tailored MMR testing. Lately, it has been shown that dMMR TNBC preferentially show high stromal T-cell predominant tumor-infiltrating lymphocytes (TILs) and higher expression of PD-L1 and CD8 than those with an MMR proficient status $(4,24)$. In another study, patients with TILs-high TNBC revealed an inverse correlation between MLH1 and PD-L1 expression in stromal immune cells (25). As pointed by Ren et al., large multicentric cohorts are needed improve our understanding of the relationship between MMR and the other actionable biomarkers in TNBC.

\section{CURRENTLY AVAILABLE TESTING METHODS AND GUIDELINES}

What we know so far is that MMR data in TNBC may vary according to the employed testing method, such as IHC for the four MMR proteins, MSI PCR, and next-generation sequencing

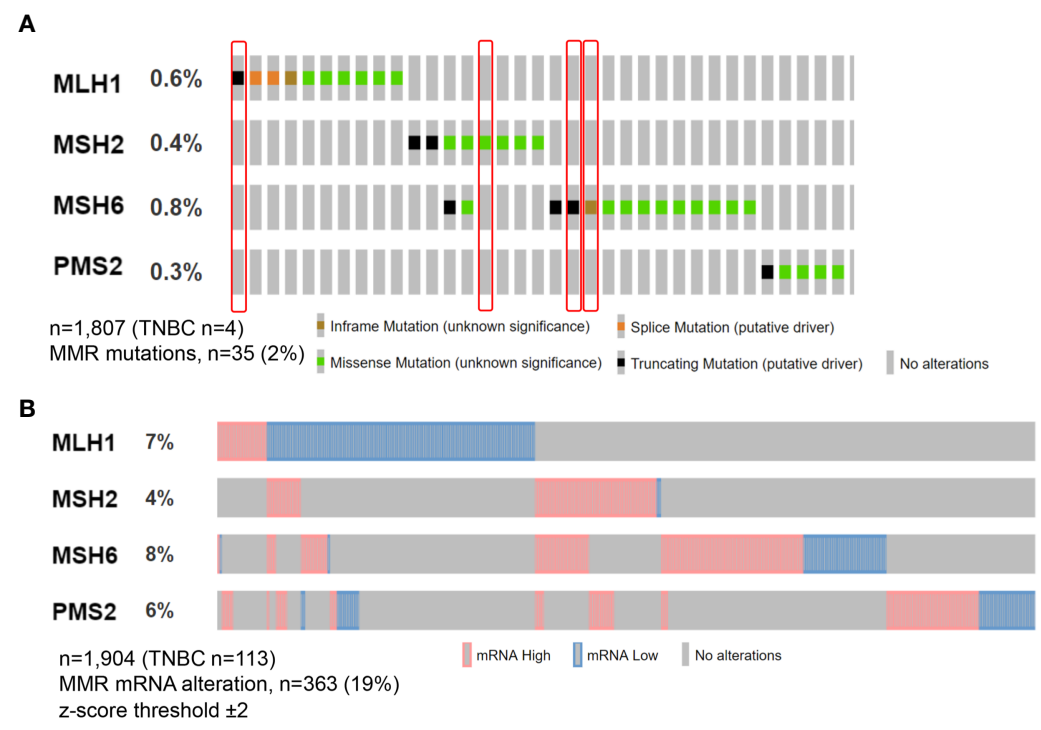

FIGURE 1 | Oncoprint visualization of molecular alterations in the MMR genes in triple-negative breast cancer. Alterations across all breast cancer subtypes are color-coded on the basis of the legends on the bottom. Each column represents a sample, each row an MMR gene. Somatic MMR mutations (A) are seen in 35 $(2 \%)$ of queried patients from MSK study with the majority showing missense mutation, while MMR mRNA alterations (B) in $n=363$ (19\%) from METABRIC study. Tumors included in this analysis have been retrieved from cbioportal.org. 
(NGS) (26). Among these, IHC is usually employed as a first-line testing method due to its reliability, cost-effectiveness, and large availability $(8,27)$. Lately, we proposed phosphatase and tensin homolog (PTEN) as a complementary biomarker in breast cancer, as its wild-type expression by IHC had a $100 \%$ positive predictive value for MMR proficiency in several subtypes, including TNBC (16). MSI analysis using mononucleotide markers, also employed by Ren et al., is a highly sensitive method, albeit not specific for breast cancer (28-31). Given that NGS-based panels can screen a larger number of microsatellite loci compared to RT-PCR and allow for the simultaneous identification of other actionable genetic alterations, this technology is currently gaining momentum in cancers with lower MSI-H/dMMR frequency, such as TNBC (32-35). Regrettably, all these methods are generally molded on those approved for the archetypal Lynch syndrome tumors, where MSI occurs way more frequently than in TNBC (colorectal cancer predominantly) (36). To ensure optimal specificity and sensitivity in breast cancer, these diagnostic strategies might need to be re-developed or at least re-validated.

\section{REFERENCES}

1. Pećina-Šlaus N, Kafka A, Salamon I, Bukovac A. Mismatch Repair Pathway, Genome Stability and Cancer. Front Mol Biosci (2020) 7:122-. doi: 10.3389/ fmolb.2020.00122

2. Barroso-Sousa R, Jain E, Cohen O, Kim D, Buendia-Buendia J, Winer E, et al. Prevalence and Mutational Determinants of High Tumor Mutation Burden in Breast Cancer. Ann Oncol (2020) 31(3):387-94. doi: 10.1016/j.annonc.2019.11.010

3. Ren X -Y, Song Y, Wang J, Chen L-Y, Pang J-Y, Zhou L-R, et al. Mismatch Repair Deficiency and Microsatellite Instability in Triple-Negative Breast Cancer: A Retrospective Study of 440 Patients. Front Oncol (2021) 11(368). doi: $10.3389 /$ fonc. 2021.570623

4. Wen YH, Brogi E, Zeng Z, Akram M, Catalano J, Paty PB, et al. DNA Mismatch Repair Deficiency in Breast Carcinoma: A Pilot Study of TripleNegative and Non-Triple-Negative Tumors. Am J Surg Pathol (2012) 36 (11):1700-8. doi: 10.1097/PAS.0b013e3182627787

5. Fusco N, Lopez G, Corti C, Pesenti C, Colapietro P, Ercoli G, et al. Mismatch Repair Protein Loss as a Prognostic and Predictive Biomarker in Breast Cancers Regardless of Microsatellite Instability. JNCI Cancer Spectr (2018) 2 (4):pky056. doi: 10.1093/jncics/pky056

6. Dasgupta H, Islam S, Alam N, Roy A, Roychoudhury S, Panda CK. Hypomethylation of Mismatch Repair Genes MLH1 and MSH2 Is Associated With Chemotolerance of Breast Carcinoma: Clinical Significance. J Surg Oncol (2019) 119(1):88-100. doi: 10.1002/jso.25304

7. Punturi NB, Seker S, Devarakonda V, Mazumder A, Kalra R, Chen CH, et al. Mismatch Repair Deficiency Predicts Response to HER2 Blockade in HER2Negative Breast Cancer. Nat Commun (2021) 12(1):2940. doi: 10.1038/ s41467-021-23271-0

8. Corti C, Sajjadi E, Fusco N. Determination of Mismatch Repair Status in Human Cancer and Its Clinical Significance: Does One Size Fit All? Adv Anat Pathol (2019) 26(4):270-9. doi: 10.1097/PAP.0000000000000234

9. Mittendorf EA, Zhang $\mathrm{H}$, Barrios $\mathrm{CH}$, Saji S, Jung KH, Hegg R, et al. Neoadjuvant Atezolizumab in Combination With Sequential Nab-Paclitaxel and Anthracycline-Based Chemotherapy Versus Placebo and Chemotherapy in Patients With Early-Stage Triple-Negative Breast Cancer (Impassion031): A Randomised, Double-Blind, Phase 3 Trial. Lancet (2020) 396(10257):1090100. doi: 10.1016/S0140-6736(20)31953-X

10. Schmid P, Cortes J, Pusztai L, McArthur H, Kümmel S, Bergh J, et al. Pembrolizumab for Early Triple-Negative Breast Cancer. N Engl J Med (2020) 382(9):810-21. doi: 10.1056/NEJMoa1910549

\section{CONCLUSION}

The diagnosis and treatment of TNBC have remarkably progressed during the recent decades, yet many patients develop resistance to pharmacotherapy and die of this disease. The pathological identification of dMMR TNBC, albeit promising, has proven to be tremendously difficult due to the constraints of the existing methods and the scarcity of research. The study by Ren et al. represents another step forward in the discussion on the clinical utility of MMR testing in breast cancer. Further translational research studies and clinical trials encompassing tumor-specific guidelines for analytical and preanalytical phases are warranted to improve the characterization of the MMR status in TNBC.

\section{AUTHOR CONTRIBUTIONS}

All the authors equally participated in the writing and reviewing of the paper. All authors contributed to the article and approved the submitted version.

11. Gianni L, Huang C-S, Egle D, Bermejo B, Zamagni C, Thill M, et al. Abstract GS3-04: Pathologic Complete Response (Pcr) to Neoadjuvant Treatment With or Without Atezolizumab in Triple Negative, Early High-Risk and Locally Advanced Breast Cancer. Neotripapdl1 Michelangelo Randomized Study. Cancer Res (2020) 80(4 Supplement):GS3-04. doi: 10.1158/15387445.SABCS19-GS3-04

12. Mills AM, Dill EA, Moskaluk CA, Dziegielewski J, Bullock TN, Dillon PM. The Relationship Between Mismatch Repair Deficiency and PD-L1 Expression in Breast Carcinoma. Am J Surg Pathol (2018) 42(2):183-91. doi: 10.1097/PAS.0000000000000949

13. Pereira B, Chin SF, Rueda OM, Vollan HK, Provenzano E, Bardwell HA, et al. The Somatic Mutation Profiles of 2,433 Breast Cancers Refines Their Genomic and Transcriptomic Landscapes. Nat Commun (2016) 7:11479. doi: 10.1038/ ncomms11479

14. Anurag M, Ellis MJ, Haricharan S. DNA Damage Repair Defects as a New Class of Endocrine Treatment Resistance Driver. Oncotarget (2018) 9 (91):36252-3. doi: 10.18632/oncotarget.26363

15. Cheng AS, Leung SCY, Gao D, Burugu S, Anurag M, Ellis MJ, et al. Mismatch Repair Protein Loss in Breast Cancer: Clinicopathological Associations in a Large British Columbia Cohort. Breast Cancer Res Treat (2020) 179(1):3-10. doi: 10.1007/s10549-019-05438-y

16. Lopez G, Noale M, Corti C, Gaudioso G, Sajjadi E, Venetis K, et al. PTEN Expression as a Complementary Biomarker for Mismatch Repair Testing in Breast Cancer. Int J Mol Sci (2020) 21(4):1461. doi: 10.3390/ijms21041461

17. Lopez G, Fusco N. RE: Mismatch Repair Protein Loss in Breast Cancer: Clinicopathological Associations in a Large British Columbia Cohort. Breast Cancer Res Treat (2020) 180:265-6. doi: 10.1007/s10549-020-05530-8

18. Helleday T, Eshtad S, Nik-Zainal S. Mechanisms Underlying Mutational Signatures in Human Cancers. Nat Rev Genet (2014) 15(9):585-98. doi: $10.1038 / \mathrm{nrg} 3729$

19. Sajjadi E, Venetis K, Piciotti R, Invernizzi M, Guerini-Rocco E, Haricharan S, et al. Mismatch Repair-Deficient Hormone Receptor-Positive Breast Cancers: Biology and Pathological Characterization. Cancer Cell Int (2021) 21(1):266. doi: 10.1186/s12935-021-01976-y

20. Viale G, Trapani D, Curigliano G. Mismatch Repair Deficiency as a Predictive Biomarker for Immunotherapy Efficacy. BioMed Res Int (2017) 2017:4719194. doi: $10.1155 / 2017 / 4719194$

21. Boyiadzis MM, Kirkwood JM, Marshall JL, Pritchard CC, Azad NS, Gulley JL. Significance and Implications of FDA Approval of Pembrolizumab for Biomarker-Defined Disease. J Immunother Cancer (2018) 6(1):35. doi: 10.1186/s40425-018-0342-x 
22. Subbiah V, Kurzrock R. The Marriage Between Genomics and Immunotherapy: Mismatch Meets Its Match. Oncologist (2019) 24(1):1-3. doi: 10.1634/theoncologist.2017-0519

23. Haricharan S, Bainbridge MN, Scheet P, Brown PH. Somatic Mutation Load of Estrogen Receptor-Positive Breast Tumors Predicts Overall Survival: An Analysis of Genome Sequence Data. Breast Cancer Res Treat (2014) 146 (1):211-20. doi: 10.1007/s10549-014-2991-x

24. Hou Y, Nitta H, Parwani AV, Li Z. PD-L1 and CD8 Are Associated With Deficient Mismatch Repair Status in Triple-Negative and HER2-Positive Breast Cancers. Hum Pathol (2019) 86:108-14. doi: 10.1016/j.humpath.2018.12.007

25. Horimoto Y, Thinzar Hlaing M, Saeki H, Kitano S, Nakai K, Sasaki R, et al. Microsatellite Instability and Mismatch Repair Protein Expressions in Lymphocyte-Predominant Breast Cancer. Cancer Sci (2020) 111:2647-54. doi: $10.1111 /$ cas. 14500

26. Venetis K, Sajjadi E, Haricharan S, Fusco N. Mismatch Repair Testing in Breast Cancer: The Path to Tumor-Specific Immuno-Oncology Biomarkers. Trans Cancer Res (2020) 9(7):4060-4. doi: 10.21037/tcr-20-1852

27. Shia J. The Diversity of Tumours With Microsatellite Instability: Molecular Mechanisms and Impact Upon Microsatellite Instability Testing and Mismatch Repair Protein Immunohistochemistry. Histopathology (2020) 78:485-97. doi: 10.1111/his.14271

28. Cicek MS, Lindor NM, Gallinger S, Bapat B, Hopper JL, Jenkins MA, et al. Quality Assessment and Correlation of Microsatellite Instability and Immunohistochemical Markers Among Population- and Clinic-Based Colorectal Tumors Results From the Colon Cancer Family Registry. J Mol Diagn (2011) 13(3):271-81. doi: 10.1016/j.jmoldx.2010.12.004

29. Bai H, Wang R, Cheng W, Shen Y, Li H, Xia W, et al. Evaluation of Concordance Between Deficient Mismatch Repair and Microsatellite Instability Testing and Their Association With Clinicopathological Features in Colorectal Cancer. Cancer Manag Res (2020) 12:2863-73. doi: 10.2147/ CMAR.S248069

30. Zeinalian M, Hashemzadeh-Chaleshtori M, Salehi R, Emami MH. Clinical Aspects of Microsatellite Instability Testing in Colorectal Cancer. Advanced Biomed Res (2018) 7:28-. doi: 10.4103/abr.abr_185_16

31. De Craene B, Van de Velde J, Rondelez E, Vandenbroeck L, Peeters K, Vanhoey T, et al. Detection of Microsatellite Instability (MSI) in Colorectal Cancer Samples With a Novel Set of Highly Sensitive Markers by Means of the Idylla MSI Test Prototype. J Clin Oncol (2018) 36(15_suppl):e15639-e. doi: 10.1200/JCO.2018.36.15_suppl.e15639
32. Bonneville R, Krook MA, Chen H-Z, Smith A, Samorodnitsky E, Wing MR, et al. Detection of Microsatellite Instability Biomarkers via Next-Generation Sequencing. Methods Mol Biol (Clifton NJ) (2020) 2055:119-32. doi: 10.1007/ 978-1-4939-9773-2_5

33. Middha S, Zhang L, Nafa K, Jayakumaran G, Wong D, Kim HR, et al. Reliable Pan-Cancer Microsatellite Instability Assessment by Using Targeted NextGeneration Sequencing Data. JCO Precis Oncol (2017) 2017:PO.17.00084. doi: 10.1200/PO.17.00084

34. Albayrak A, Garrido-Castro AC, Giannakis M, Umeton R, Manam MD, Stover EH, et al. Clinical Pan-Cancer Assessment of Mismatch Repair Deficiency Using Tumor-Only, Targeted Next-Generation Sequencing. JCO Precis Oncol (2020) 4):1084-97. doi: 10.1200/PO.20.00185

35. Angerilli V, Galuppini F, Pagni F, Fusco N, Malapelle U, Fassan M. The Role of the Pathologist in the Next-Generation Era of Tumor Molecular Characterization. Diagnostics (2021) 11(2):339. doi: 10.3390/diagnostics 11020339

36. Kucab JE, Zou X, Morganella S, Joel M, Nanda AS, Nagy E, et al. A Compendium of Mutational Signatures of Environmental Agents. Cell (2019) 177(4):821-36.e16. doi: 10.1016/j.cell.2019.03.001

Conflict of Interest: NF has received honoraria for consulting, advisory role, and/ or speaker bureau from Merck Sharp \& Dohme (MSD), Boehringer Ingelheim, and Novartis.

The remaining authors declare that the research was conducted in the absence of any commercial or financial relationships that could be construed as a potential conflict of interest.

Publisher's Note: All claims expressed in this article are solely those of the authors and do not necessarily represent those of their affiliated organizations, or those of the publisher, the editors and the reviewers. Any product that may be evaluated in this article, or claim that may be made by its manufacturer, is not guaranteed or endorsed by the publisher.

Copyright (๑) 2021 Venetis, Fusco and Sajjadi. This is an open-access article distributed under the terms of the Creative Commons Attribution License (CC BY). The use, distribution or reproduction in other forums is permitted, provided the original author(s) and the copyright owner(s) are credited and that the original publication in this journal is cited, in accordance with accepted academic practice. No use, distribution or reproduction is permitted which does not comply with these terms. 